\title{
Sprachverstehen im Kontext: Bildgebende Studien zu Kohärenz und Pragmatik
}

\author{
E. C. Ferstl ${ }^{1,2}$ \\ D. Y. von Cramon ${ }^{1,2}$
}

\author{
Understanding of Speech in Context: Imaging Studies on Coherence \\ and Pragmatism
}

\section{Zusammenfassung}

Kommunikation im Alltag erfordert immer Interpretationen, die neben der wörtlichen Bedeutung des gerade Gesagten oder Gehörten das Hintergrundwissen, die Zielsetzung oder die Kontextinformation berücksichtigen. Neben den Aphasien werden auch so genannte nicht-aphasische Kommunikationsstörungen beschrieben, die erst in einer komplexen Kommunikationssituation manifest werden. In der Neurowissenschaft werden pragmatische und kontextuelle Aspekte des Sprachverstehens oft mit rechts-hemisphärischen Funktionen oder mit Exekutivfunktionen in Verbindung gebracht. Bildgebende Studien bestätigen für alle Aspekte der Sprachverarbeitung im Kontext sowohl präfrontale als auch rechtsseitige Beteiligung. Insbesondere der anteriore Temporallappen und der fronto-mediane Kortex spielen eine spezifische Rolle für die inhaltliche Interpretation und Verknüpfung von aufeinanderfolgenden Äußerungen. Die klinische Diagnose der kommunikativen Kompetenz von hirngeschädigten Patienten sollte deshalb zusätzlich zu einer Aphasiediagnositk immer eine Bewertung von Textverständnis und Diskursproduktion beinhalten.

\begin{abstract}
Communicative compentence requires the interpretation of utterances in light of background knowledge, comprehension goal and context information. In addition to aphasic language disorders so-called non-aphasic communication deficits have been described. These deficits manifest themselves whenever non-literal meanings are relevant or when the global context or structure needs to be derived. In neuropsychological research the focus has been on the frontal lobes and the right hemisphere. Recent neuroimaging studies have accumulated evidence for both of these brain regions to be involved during most aspects of complex language comprehension. In particular the anterior temporal lobes and the fronto-medial cortex play a specific role for the integration and interpretation of verbal information. Clinical diagnosis of brain damaged patients should therefore include an assessment of contextual language processing in addition to the evaluation of aphasic language deficits.
\end{abstract}

\section{Einleitung}

Kommunikative Kompetenz ist eine wichtige Voraussetzung für den Rehabilitationserfolg nach Hirnschädigung [1]. In den meisten beruflichen Feldern sind sprachliche Fähigkeiten unerlässlich, und die Nutzung neuerer Medien in unserer Informationsgesellschaft erfordert die ständige Integration von sprachlichen
Äußerungen mit Kontext, Weltwissen oder nicht-sprachlicher Information. Diese komplexen kognitiven Leistungen erfordern das Zusammenwirken einer Vielzahl von Prozessen. In der Psycholinguistik und Neurowissenschaft werden daher sprachsystematische Prozesse auf der Wort- und Satzebene von strukturierenden und interpretativen Prozessen auf der Textebene unterschieden [2].

Institutsangaben

${ }^{1}$ Max-Planck-Institut für Kognitions- und Neurowissenschaften, Leipzig

${ }^{2}$ Tagesklinik für kognitive Neurologie, Universität Leipzig

Korrespondenzadresse

Dr. Evelyn C. Ferstl - Max-Planck-Institut für Kognitions- und Neurowissenschaften - Stephanstr. $1 \mathrm{~A}$. 04103 Leipzig · E-mail: ferstl@cbs.mpg.de

Bibliografie

Sprache · Stimme · Gehör 2005; 29: 130-138 • @ Georg Thieme Verlag KG Stuttgart · New York DOI 10.1055/s-2005-873120

ISSN 0342-0477 
Auch die Störungsbilder nach erlittener Hirnschädigung bestätigen die Bedeutung der Textebene für die Kommunikation. Vor allem nach Schädel-Hirn-Trauma und nach rechtsseitigen Hirnläsionen werden so genannte nicht-aphasische Kommunikationsstörungen beobachtet $[3,4]$, die im englisch-sprachigen Raum auch mit Cognitive Communication Impairment bezeichnet werden [5]. Symptome wurden vor allem für die Sprachproduktion beschrieben. Sie reichen von reduzierter, inhaltlich unvollständiger oder überschüssiger, weitschweifender Sprache, über unstrukturierte, inkohärente Redeweise, das Vernachlässigen von Kontextinformation oder Vorwissen des Hörers, bis zu sozial unangemessenem sprachlichen Verhalten. Um diese durch so vielfältige und teils disparate Symptome charakterisierten Störungen diagnostizieren zu können, ist es wichtig, spezifischere Kenntnisse über die funktionelle Neuroanatomie von pragmatischer und kontextueller Sprachverarbeitung zu gewinnen.

In diesem Artikel wollen wir einen Überblick über die bildgebende Forschung zu diesem Thema geben. Dazu führen wir im ersten Abschnitt einige Teilaspekte der Textverarbeitung ein. Nach einer Skizze von Erklärungsmodellen gehen wir dann über zu Studien, die mit bildgebenen Verfahren, insbesondere Positronen-Emissions-Tomographie (PET) und funktioneller Magnetresonanztomographie (fMRT) der Frage nach der funktionellen Neuroanatomie von pragmatischer und kontextueller Sprachverarbeitung nachgehen.

\section{Einige Begriffe aus der Psycholinguistik}

Bevor wir uns neuropsychologischen und neurowissenschaftlichen Forschungsergebnissen zuwenden, ist es nötig, die verschiedenen Teilaspekte von Sprachinterpretation im Kontext spezifischer zu umgrenzen [vgl. 2]. Im Folgenden führen wir drei teils überlappende, aber in der Psycholinguistik unterschiedene Bereiche ein: die Kohärenz, das Situationsmodell, und pragmatische Aspekte.

\section{Kohärenz}

Der wichtigste Teilprozess des Sprachverstehens im Kontext ist die Verknüpfung von aufeinanderfolgenden Äußerungen oder Sätzen zu einem kohärenten Ganzen. Diese Inferenzprozesse benötigen nicht nur die wörtliche Formulierung der relevanten Inhalte, sondern meist auch das Einbeziehen von Weltwissen oder kontextuellem Vorwissen. Ein einfaches Beispiel ist das Satzpaar: Marlene ist zum Geburtstag eingeladen. Sie schlachtet ihr Sparschwein. Der - für uns scheinbar offensichtliche Zusammenhang basiert nicht auf einer assoziativen Überlappung der Inhaltswörter. Auch die lexikalische Information, die beiden Pronomen sie und ihr (auch Kohäsionsmarkierungen genannt), gibt nicht ausreichend Aufschluss über den inhaltlichen Zusammenhang der Sätze. Nur durch das Wissen über unsere kulturellen Gepflogenheiten wird der Sachverhalt verständlich, dass Marlene wohl mit Ihrem Ersparten ein Geschenk für das Geburtstagskind kaufen möchte. Dieser relativ einfache Sachverhalt illustriert, dass gleichzeitig eine Reihe unterschiedlicher Inferenzen gezogen werden können. Zu der kohärenzstiftenden Brücken-Inferenz kommen elaborative Inferenzen (z. B., „Marlene ist ein kleines Mädchen“) und finale Inferenzen über die Ziele der Protagonistin („Marlene plant, ein Geschenk zu kaufen“). Diese verschiedenen Inferenzarten wurden in der Psycholinguistik als qualitativ unterschiedlich bewertet [6,7].
Auf globaler Ebene besteht die Kohärenzbildung darin, eine Struktur des Gesamttextes abzuleiten [2,8]. Diese so genannte Makrostruktur, die man sich auch als Gliederung, Zusammenfassung oder Inhaltsverzeichnis vorstellen kann, benötigt einerseits die schon genannten lokalen Inferenzen, aber andererseits auch Inferenzen, die den zeitlichen Ablauf, oder die Gewichtung von Informationen berücksichtigt. So werden die Leser dieses Artikels u. U. noch erinnern, dass wir uns jetzt gerade im Abschnitt über Kohärenz befinden - und darauf warten, dass in einem der nächsten Abschnitte auf das Situationsmodell eingegangen wird. Diese explizite Gliederungshilfe erleichtert also die Strukturierung, ist aber durchaus nicht für jeden Text oder jedes Gespräch typisch. Die Hörerin oder der Leser müssen sich also durch Reduzierung, Verallgemeinerung, oder Sequenzierung - also der Anwendung von Makroregeln - aktiv an der Strukturierung beteiligen. Umgekehrt gilt für die Sprachproduktion, dass eine ständige Auswahl, Strukturierung und Gewichtung nötig ist, um die geplanten Inhalte auch angemessen zu vermitteln. Wenn z. B. eine Geschichte in einer von der chronologischen Reihenfolge abweichenden Weise erzählt wird, kann die Hörerin nicht so leicht folgen. Dann müssen die zeitlichen Sprünge explizit angezeigt werden, wie es etwa bei Rückblenden im Film üblich ist.

\section{Situationsmodell}

Das Situationsmodell ist ein mentales Modell des Gehörten oder Gelesenen, also eine globale Vorstellung davon, worum es in einem Text geht [9-11]. Hier wird von der wörtlichen Formulierung abstrahiert. Die aktuellen Äußerungen werden mit Vorwissen und Kontextinformation integriert, so dass die resultierende Repräsentation für jeden Rezipienten unterschiedlich ausfällt. Für Erzählungen wurde gezeigt, dass Informationsdimensionen, wie das Wo, Wie, Wann oder Wer der Geschichte im Situatonsmodell enkodiert werden [12]. Die Repräsentation kann auch nicht-verbal sein, wenn der jeweilige Inhalt dies nahelegt. So wird z. B. das Lesen eines Artikel über ein Fußballspiel bei einem Fußballfan sofort eine visuelle Vorstellung des Spielfelds beinhalten, oder die Nervosität wieder aufleben lassen, die er während des Spiels empfand. Ein Leser ohne spezielles Interesse am Thema wird dagegen eine sparsamere, möglicherweise sprachlich-propositionalere Repräsentation erstellen, die näher am explizit formulierten Text bleibt. Leser und Hörerinnen nutzen das Situationsmodell, um Texte zu interpretieren, und ihre Inhalte langfristig zu erinnern, weiterzugeben, und in das deklarative Weltwissen einzubinden [13].

\section{Pragmatische Aspekte}

Ein wichtiger Bereich von Kommunikation sind nicht-wörtliche Interpretationen, wie sie im Alltag mühelos und effektiv eingesetzt werden. Ob es um geschäftliche Verhandlungen oder Witze erzählen geht - ohne die flexible und kreative Nutzung von Sprache wäre soziales Zusammenleben nicht vorstellbar. Wie das Beispiel im vorigen Abschnitt zeigte, lassen sich kognitive und pragmatische Aspekte nicht trennen. Das Situationsmodell kann durchaus Wissen integrieren, aber auch affektive Begleitmusik enthalten. In der Linguistik beschreibt die Pragmatik die Faktoren, die in Abhängigkeit von der kommunikativen Situation und Intention die Sprachauswahl und die Interpretation bestimmen [14]. Der gleiche Sachverhalt kann in vielerlei Weise ausgedrückt werden, und wird durch die spezielle Wahl einer Sprachebene, einer Formulierung, oder einer Betonung beim Hörer unter- 
schiedliche Interpretationen und Reaktionen hervorrufen. So wird z. B. die gleiche Äußerung „schön warm hier!“ je nach Situation unterschiedlich interpretiert: Ironie, wenn die Heizung kaputt ist, Erfolgsmeldung für den Heizungsmonteur nach der Reparatur, Urlaubsgeplänkel am Sandstrand, oder im überfüllten Klassenzimmer die Aufforderung, ein Fenster zu öffnen.

Neben paraverbalen Ausdrucksmitteln (wie z. B. Prosodie, Mimik oder Gestik) spielen vor allem soziale Konventionen (z. B. Höflichkeit, Dialogregeln), und der situative Kontext eine Rolle. Sowohl Sprecherin als auch Hörer müssen diese Regeln zur Verfügung haben und anwenden. Wichtig ist, dass eine angemessene Nutzung der pragmatischen Faktoren oft impliziert, dass die wörtliche Lesart mit der Kommunikationsintention nicht übereinstimmt. Ausdrucksformen, die daraus entstehen, sind z. B. Metaphern und Idiome, Sprichwörter, indirekte Aufforderungen, Ironie, Sarkasmus und verbalen Humor [vgl. 15].

Bevor wir näher auf die Ergebnisse aus der Bildgebung eingehen, soll im nächsten Abschnitt eine kurze Übersicht über die neuropsychologischen Theorien zum Sprachverstehen im Kontext gegeben werden [s. 16].

\section{Neuropsychologische Theorien der Sprachverarbeitung im Kontext}

Wie schon zu erwarten, sind die neuropsychologischen Theorien über kontextuelle Sprachnutzung genauso vielfältig, wie die Aspekte, die sie beschreiben sollen. Vorherrschend ist dabei die Sicht, dass die rechte, oder richtiger, die nicht sprach-dominante Hemisphäre, diese Prozesse implementiert. Es gibt jedoch unterschiedliche Konzeptionen darüber, wie diese so genannte RHHypothese funktionell zu spezifizieren ist. Die umfassendste Theorie geht in Analogie zur visuellen Wahrnehmung davon aus, dass die linke Hemisphäre lokale Sprachprozesse auf Wortund Satzebene implementiert, während die rechte für die globalen, also interpretativen und integrativen Prozesse auf Text- und pragmatischer Ebene verantwortlich ist [17].

Bei der Ressourcen-Theorie [18] wird ebenfalls davon ausgegangen, dass die „normale“ Sprachverarbeitung von der linken Hemisphäre übernommen wird. Die Rolle der rechten Hemisphäre besteht jedoch darin, im Fall von erhöhter Komplexität oder Schwierigkeit unterstützend einzugreifen. Eine empirische Überprüfung dieser Theorie ist oft dadurch erschwert, dass sie eine genaue Definition von Komplexität oder Schwierigkeit voraussetzen würde. Eine direkte Übertragung auf die in der Psycholinguistik definierten Teilprozesse von der Wort- über die Satz- zur Textebene ist nicht unmittelbar möglich; das Erstellen eines Situationsmodells auf Textebene etwa ist nicht notwendigerweise schwieriger, als die grammatikalische Analyse auf Satzebene.

Eine dritte, sehr einflussreiche Theorie ist die so genannte Coarse-Coding-Hypothese [19]. Diese semantisch basierte Theorie wurde entwickelt, um Inferenzprozesse zu erklären. Sie geht davon aus, dass in der rechten Hemisphäre - und dort wohl vor allem im Temporallappen - weite semantische Felder aktiviert werden, durch deren Überlappung eine Brückeninferenz gefunden werden kann. In dem obigen Beispiel etwa aktiviert das Inhaltswort Geburtstag neben Geschenk auch kaufen und damit Geld; dieser letzte Begriff ist natürlich auch im semantischen
Feld von Sparschwein enthalten. Diese kontext-relevante Brücke erfordert die initial unselektierte Aktivierung einer Vielzahl von Inhalten. Die Auswahl, also Deaktivierung der irrelevanten Inhalte soll von der linken Hemisphäre vorgenommen werden [19]. Diese Konzeptualisierung ist dem kognitionspsychologisch motivierten Konstruktions-Integrations-Modell ähnlich [20], das ohne neuroanatomische Hypothesen ebenfalls ein Wechselspiel von breiter Aktivierung (Konstruktion) und nachfolgender Selektion (Integration) postuliert.

Schließlich beziehen sich viele Autoren auf die pragmatisch-affektiven Funktionen der rechten Hemisphäre [15]. Die Hypothese hier ist wieder, dass die linke Hemisphäre primär für die Sprachverarbeitung zuständig ist, dass aber paraverbale Informationen, wie z. B. die Prosodie, oder sozial-interpretative Prozesse von der rechten Hemisphäre verarbeitet werden sollen. Da in der neuropsychologischen Forschung häufig eine Vermischung der kognitiven und pragmatischen Aspekte der Kontextnutzung vorliegt [21], lässt sich diese Theorie vor allem durch die Überprüfung nichtverbaler Entsprechungen dieser Funktionen testen.

Eine alternative Sichtweise zu den Lateralisierungstheorien stellt den Beitrag von Frontalhirnfunktionen in den Vordergrund $[3,4,22,23]$. Die Beteiligung des präfrontalen Kortex wird einerseits in einer Vielzahl von klinischen Studien deutlich, die vor allem für Schädel-Hirn-Trauma-Patienten aphasische Sprachstörungen nachwiesen. Andererseits ergibt sie sich direkt aus der Definition der Teilaspekte. Die kognitiven Aspekte, wie z. B. Strukturierung, Inhibition irrelevanter Inhalte, zielgerichteter Wissensabruf oder Kontextnutzung, sind Teilfunktionen des Komplexes der Exekutivfunktionen - die sicher im präfrontalen Kortex realisiert werden. Eine einfache Reduktion von nicht-aphasischen Kommunikationsstörungen auf „exekutive“ Prozesse, wie z. B. Inhibtion oder Aufmerksamkeit, scheint jedoch nicht zu gelingen [24]. Kommunikationsfähigkeiten leisten einen unabhängigen Beitrag zur Abgrenzung von Störungen des Frontalhirns. Auch für die pragmatisch-affektiven Komponenten kann man eine Beteiligung des Frontalhirns annehmen, allerdings in anderen Regionen. Insbesondere der orbito-frontale und ventro-mediane präfrontale Kortex wird mit sozialer Anpassung und affektiver Verarbeitung in Verbindung gebracht $[25,26]$.

Die RH-Hypothese und die Theorien, die Frontalhirnfunktionen in den Vordergrund stellen, sind natürlich nicht inkompatibel. Zum einen werden in vielen Studien über rechts-hemisphärische Kommunikationsstörungen auch Patienten eingeschlossen, deren Läsionen in den Frontallappen hinein reichen [27-29]. Andererseits basieren viele der empirischen Befunde über Frontalhirnbeteiligung auf den Ergebnissen von Schädel-Hirn-TraumaPatienten, deren Läsionen meist nicht eindeutig lateralisiert sind. Umschriebene, kleine Läsionen können jedoch oft gut kompensiert werden, wie die klinische Erfahrung zeigt. Daher ist eine genauere Eingrenzung dieser Theorien mit Hilfe von neuropsychologischen Läsionsstudien schwierig.

\section{Bildgebende Studien}

Mit der Methode der funktionellen Bildgebung können wir indirekt über die begleitende hämodynamische Reaktion beobach- 
ten, welche Hirnareale bei der Sprachverarbeitung im Kontext beteiligt sind. Im Gegensatz zu Studien auf Wortebene ist die Literatur zu diesem Thema derzeit noch überschaubar [16,30-32]. Die wichtigsten Ergebnisse zum Textverstehen fassen wir im Folgenden zusammen. Studien zur Sprachproduktion werden dabei nicht berücksichtigt [s. 32]

\section{Das erweiterte Sprachnetzwerk}

Der erste Schritt, um die Hirnareale zu identifizieren, welche bei der Sprachverarbeitung im Kontext eine Rolle spielen, ist Aktivierungsmuster zu betrachten, die beim Hören oder Lesen von zusammenhängendem Text entstehen. Interessanterweise sind die Ergebnisse sehr konsistent, unabhängig von der Modalität (visuell oder auditorisch), von der Textlänge (Satzinterpretation oder zusammenhängender Text), oder der Aufgabenstellung (z. B. passives Zuhören oder Gedächtnis-Instruktion). Das oft replizierte Muster zeigt ein bilaterales, links-hemisphäriell dominantes Netzwerk, das vor allem fronto-temporale Regionen umfasst [s. 16]. Abb.1 zeigt ein Beispiel für dieses typische Netzwerk aus einer unserer Studien [33].

Im Temporallappen sind Aktivierungen entlang des Sulcus Temporalis Superior berichtet worden, die neben dem posterioren Sprachareal und dem benachbarten temporo-parietalen Übergang bis in den anterioren Temporallappen bzw. den Temporalpol reichen. Spezifisch für die Sprachverarbeitung im Kontext scheinen dabei vor allem die anterioren Anteile.

Im Frontallappen beinhalten die Aktivierungen sowohl inferiore Regionen, die in der linken Hemisphäre mit phonologischer, semantischer und syntaktischer Verarbeitung in Verbindung stehen (BA 44/45), als auch dorsale Regionen im mittleren frontalen Gyrus, deren Funktion wohl eher mit Arbeitsgedächtnis und der Aufgabensteuerung zu tun haben (BA 9/46). Eine Beteiligung die-

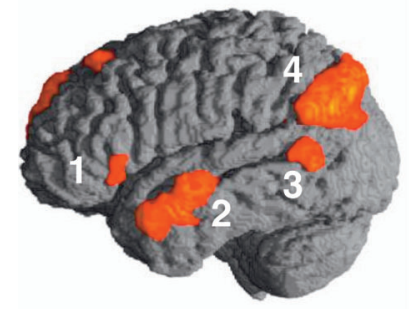

links-latera

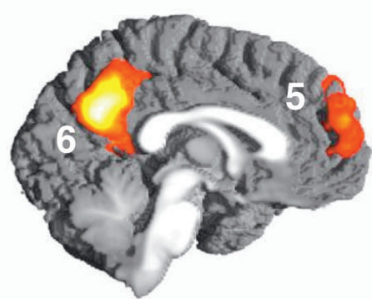

links-median
Abb. 1 Ein Beispiel für ein typisches Aktivierungsmuster für Sprachverstehen im Kontext (nach Ferstl \& von Cramon, 2002). Satzpaare wurden über Kopfhörer vorgespielt und die Kontrollbedingung bestand aus sprachähnlichen Nichtwörtern. In der linken Hemisphäre zeigen sich laterale Aktivierungen entlang des superioren temporalen Sulcus, von denen die anteriore deutlicher ist (2; BA 21/38), als die posteriore (3; BA 21/22). In der rechten Hemisphäre (hier nicht gezeigt), ist ebenfalls der anteriore Temporallappen aktiviert. Außerdem ist eine Region im temporo-parietalen Übergangsbereich beteiligt (4; BA 22/39). Im lateralen präfrontalen Kortex ist hier nur eine kleine Aktivierung im inferioren frontalen Gyrus zu sehen (1; BA 45/47). Je nach Aufgabenstellung und Kontrollbedingung werden ausgedehntere dorsalere präfrontale Aktivierungen beobachtet. Die linke Medianfläche zeigt die paarweise Aktivierung des dorsalen fronto-medianen Kortex (5; BA 9/10) und des parieto-medianen Kortex (6; Präcuneus/posteriorer zingulärer Kortex; BA 23/30/31). ser letztgenannten Areale erfordert immer eine genaue Analyse der verwendeten Aufgabe und der Kontrollbedingung. Neben den lateralen präfrontalen Regionen erweisen sich frontodorsale Aktivierungen bzw. dorsale fronto-mediane Aktivierungen als wesentlich (dmPFC; BA 8/9/10) [33 - 35]. In einer ersten PET-Studie erwies sich, dass nur diese Aktivierung (BA 8) spezifisch für das Hören von zusammenhängenden Geschichten war, während anterior temporale Regionen schon beim Satzverstehen beteiligt waren [36]. Kürzlich wurde dieses Ergebnis in einer fMRI-Studie repliziert [37]. Gepaart mit der dmPFC-Aktivierung findet man häufig eine parieto-mediane Beteiligung im posterioren zingulären Kortex bzw. Präcuneus (Prec/PCC); [33,34,37,38].

Der Vollständigkeit halber muss erwähnt werden, dass sehr ähnliche Aktivierungsmuster auch aus Studien bekannt sind, in denen kontextuelle Sprachverarbeitung scheinbar keine Rolle spielt. So fanden Binder und Kollegen sowohl für eine semantische Aufgabe mit Wort-Stimuli $[39,40]$, als auch für eine Ruhebedingung im Vergleich mit einer aufmerksamkeitsbindenden Aufgabe [41] ganz ähnliche Aktivierungen. Eine Erklärung dafür könnte sein, dass die Versuchspersonen während dieser Aufgabe selbst-gesteuerte, verbale Denkvorgänge initiierten. Eine spezifischere Zuordnung der Teilareale zu funktionell definierten Teilprozessen wird somit erst durch die Untersuchung fokussierterer Kontraste möglich.

\section{Kohärenz}

In diesem Abschnitt werden Studien zusammengefasst, die kohärente, also inhaltlich zusammenhängende Texte, mit inkohärenten vergleichen. In einer ersten eigenen fMRT-Studie ließen wir Versuchspersonen einfache Satzpaare lesen, wie sie im obigen Beispiel diskutiert wurden [34]. Die Aufgabe war, ein Kohärenzurteil abzugeben, also einfach anzuzeigen, ob die beiden Sätze inhaltlich miteinander in Beziehung standen oder nicht. Die Kontrollbedingung waren unrelatierte Satzpaare, wie z. B. Christl bekam eine Fünf in Mathe. Sie schlachtete ihr Sparschwein. Für den Vergleich all dieser Satzpaare mit einer perzeptuellen nicht-sprachlichen Baseline fanden wir ein ähnliches Aktivierungs-Muster wie im vorherigen Abschnitt beschrieben. Der interessantere Vergleich, bei dem kohärente und unrelatierte Satzpaare direkt kontrastiert wurden, ergab keine zusätzlichen Aktivierungen für die nicht-kohärenten Satzpaare. Stattdessen bewirkte eine erfolgreiche Inferenz eine stärkere Beteiligung des dmPFC und des Prec/PCC. Dieses Ergebnis konnte für auditive Präsentation, für andere Instruktionen und mit anderen Satzmaterialen repliziert werden $[16,33,42,43]$. Evidenz für die Bedeutung des dmPFC für Inferenzprozesse wurde kürzlich auch für prädiktive Inferenzen berichtet [44]. Eine besondere Rolle für die rechte Hemisphäre konnte in diesen Studien nicht nachgewiesen werden.

Eine Reihe anderer Studien zur Inferenzbildung benutzten unterschiedliche Materialien und Aufgaben sowie andere Auswertemethoden. Ein direkter Vergleich mit den oben genannten Ergebnissen ist daher erschwert. Die Befunde konvergieren jedoch, da sie eine Beteiligung rechtshirniger, fronto-temporaler Regionen nahe legen. Robertson und Kollegen [45] fanden rechts-laterale inferior-frontale Aktivierung, wenn durch definite Artikel eine kohärente Interpretation nahegelegt wurde. In einer weiteren Studie wurden Inferenzen mit unterschiedlicher Distanz ver- 
glichen [46]. Satzpaare wurden präsentiert, die durch eine nahe, moderate oder weite Inferenz zu überbrücken waren. Die Autoren berichten stärkere rechts-temporale Aktivierung für die moderaten Inferenzen. Im Gegensatz dazu stieg die Aktivierung in lateralen präfrontalen Arealen beidseits mit der Schwierigkeit der Inferenzbildung an. Die Region-of-Interest (ROI) Analyse, bei der anatomisch definierte Bereiche zusammengefasst ausgewertet werden, lässt nicht erkennen, ob fronto-mediane Areale eine Rolle spielten. In einer Studie über die die Verarbeitung von Themenwechseln in kurzen Dialogen, die ja ebenfalls Kohärenzbildung erfordert, wurde ein ausgedehntes, bilaterales fronto-temporales Netzwerk beschrieben [47].

Zusammenfassend lässt sich also schlussfolgern, dass für die Kohärenzbildung frontale Areale, sowohl lateral als auch median, aber auch rechtshirnige temporale Regionen wesentlich sind. Eine Zuordnung gemäß der Aufgaben- oder Materialcharakteristika ist noch nicht möglich, da die gewählten Methoden keinen direkten Vergleich der Studien zulassen. Weitere Forschung ist nötig, um die Faktoren eingrenzen zu können, die eine Beteiligung dieser unterschiedlichen Areale wahrscheinlich machen. Vor allem ist eine qualitative Unterscheidung verschiedener Inferenzarten wünschenswert.

\section{Das Situationsmodell}

In den vorherigen Studien über Kohärenz spielt immer auch das Situationsmodell eine Rolle. Sprachliche Ausdrucksmittel und die daraus resultierenden Inferenzen sind ein wichtiger Teil der Situationsmodellbildung, aber andererseits ermöglicht oft auch erst das Vorhandensein eines geeigneten Situationsmodells, also eines Ankers in der Welt, die relevanten Inferenzen.

Drei Studien untersuchten explizit die Situationsmodellbildung während des Textverstehens. Mittels eines klassischen Paradigmas verglichen zwei Studien das Verstehen von Sprache, wenn ein Situationsmodell aufgebaut werden konnte, mit einer gleichlautenden Kontrollbedingung ohne inhaltliches Verständnis. In der Textversion werden Sätze präsentiert, wie z. B. Erst muss man alles in verschiedene Haufen sortieren. Dann kommt noch Pulver dazu... Nur wenn vorher der Titel „Wäsche Waschen“ gegeben wurde, können diese Sätze in eine kohärente Interpretation integriert werden [48]. In einer PET-Studie wurde mehr Aktivierung im rechten Temporallappen beobachtet, wenn kein Titel gegeben wurde, wenn also die Passage unzusammenhängend blieb [49]. Fronto-mediane Areale wurden in der Auswertung nicht berücksichtigt.

In einem sehr ähnlichen, aber komplexeren Experiment [38] wurde die Bildversion des Paradigmas untersucht [50]. Hier wird eine unübliche Szene auf einem Bild gezeigt, z. B. spielt ein Gitarrist eine Serenade für seine Freundin, die in einem Hochhaus wohnt. Damit sie die Musik hören kann, hängt er den Lautsprecher an einen Luftballon, der ihn vor ihr Fenster in der 5. Etage trägt. Ein vage formulierter Text beschreibt dann die Szene. Ohne das Bild gelingt es nicht, die miteinander nur lose verknüpften Inhalte in ein Situationsmodell zu integrieren. Zusätzlich zu diesen Texten - in einer Bedingung mit, in einer ohne Bild - wurden andere Geschichten präsentiert, die auch ohne Bild verständlich waren. Interessanterweise berichten die Autoren aussschließlich linksseitige und mediane Aktivierungen. Insbe- sondere waren der anteriore Temporallappen, der Präcuneus und ein fronto-medianes Areal an der Verarbeitung verständlicher Geschichten mehr beteiligt als an der unverständlicher. Diese letzte Aktivierung war jedoch ventraler lokalisiert (BA11), als die in den obigen Studien zur Kohärenz. Wie im Abschnitt über die pragmatischen Aspekte ausgeführt wird, könnte dies evtl. auch ein Nebeneffekt der Witzigkeit mancher der unüblichen Szenarien sein.

In einer unserer eigenen Studien über die Situationsmodellbildung knüpften wir an die vorherigen Experimente über Kohärenzbildung an [51]. Statt Texte zu präsentieren, für die fast keine Möglichkeit besteht, einen Sinnzusammenhang zu erstellen, nutzten wir das Inkonsistenzparadigma [52]. Hier werden in kurze Alltags-Geschichten Fehler eingestreut, die erst im Gesamtzusammenhang erkennbar werden. Ein Beispiel ist der Satz „Sarah hatte sich noch nie so traurig gefühlt", der unplausibel wird, wenn er in einer Geschichte über eine Studentin vorkommt, die ausgelassen mit all ihren Freunden eine Party feiert. Die Kontrollbedingung waren konsistente Versionen der gleichen Geschichten. Zusätzlich zu diesen emotionalen Verletzungen gab es eine zeitliche Bedingung, in der die Inkonsistenz mit zeitlicher Information aus dem Kontext zu tun hatte. Z. B. wurde zuerst erzählt, dass ein Rentner vor der Skatrunde noch zum Einkaufen wollte, aber im späteren Verlauf der Geschichte wurde diese Reihenfolge umgekehrt. Dass diese beiden Informationaspekte (Zeit und Emotion) wesentliche Dimensionen eines narrativen Situationsmodells sind, ist aus der psycholinguistischen Forschung bekannt [11,12].

Für die Auswertung der fMRT-Daten waren zwei Zeiträume relevant. Zuerst untersuchten wir, was das Zielwort („traurig“) unmittelbar beim Hören auslöst. Inkonsistente Wörter aktivierten einen kleinen Bereich im rechten anterioren Temporallappen. Interessanter war jedoch der Befund, dass emotionale Wörter im ventro-medianen präfrontalen Kortex Aktivierung auslösten. Dieses Areal wird zwar mit emotional-affektiven und sozial relevanten Prozessen in Verbindung gebracht; dass aber schon das blosse Hören einer Geschichte eine Beteiligung diesen Areals nachweisen läßt, zeigte, dass die Situationsmodellbildung domänen-spezifische Repräsentationen nutzt. Bei der zeitlichen Zielinformation sah das Muster ganz anders aus. Hier fand sich ein bilaterales fronto-parietales Netzwerk, das wohl im Zusammenhang mit erhöhten Anforderungen an Aufmerksamkeit und Gedächtnis interpretiert werden sollte. Ein inferior parietaler Teil dieses Netzwerks findet sich aber auch in nicht-verbalen Aufgaben zur Verarbeitung von quantitativer Information [53], wiederum konsistent mit der Vorstellung einer unmittelbaren Beteiligung domänen-spezifischer Areale während der Situationsmodellbildung.

Schließlich interessierte uns der Integrationsprozess, also der Zeitraum zwischen dem Hören der Zielinformation und dem Ende der Geschichte, einige Sätze später. Die inkonsistente Information muss ja mit der nachfolgenden Erzählung in Einklang gebracht werden. Z. B. kann man durch eine Inferenz oder eine Elaboration gut erklären, warum Sarah auf ihrer Party traurig war. Bei der verdrehten Reihenfolge ist das nicht so leicht möglich hier wird vielmehr nötig, die Gedächtnisrepräsentation nochmals zu überprüfen, um die Information als fehlerhaft klassifi- 
zieren zu können. Im Einklang mit diesen unterschiedlichen Integrations- oder Reparaturstrategien fanden wir unterschiedliche Aktivierungsmuster. Inkonsistente emotionale Geschichten lösten mehr Aktivierung im dorsalen fronto-medianen Kortex aus, dem Areal, das auch in dem Kohärenz-Experiment als für Inferenzen wesentlich identifiziert worden war. Inkonsistente zeitliche Geschichten lösten wiederum lateral präfrontale Aktivierung aus, in Arealen, die mit Gedächtnis- und Exekutivfunktionen funktionell in Verbindung gebracht werden.

Diese Studien zur Situationsmodellbildung zeigen wiederum ein differenziertes Bild. Sie bestätigen sowohl die Beteiligung von rechtshirnigen als auch von präfrontalen, insbesondere frontomedianen Regionen. Wichtig ist jedoch, dass spezifische Textinhalte eine unmittelbare Resonanz in domänen-spezifischen Arealen bewirken können, ein Ergebnis, das Aufschluss über qualitative Eigenschaften der Situationsmodellrepräsentation geben kann [vgl. 54].

\section{Pragmatik}

Die erste Studie über pragmatische Kommunikation beschäftigte sich mit Metaphern [55]. Während der Bewertung der Plausibilität von Metaphern (z. B. Paul hat den Verstand einer Gans) war der rechtsseitige präfrontale Kortex stärker aktiviert als während der Interpretation von nicht-metaphorischen Sätzen (z. B. Der Jäger benutzte den Tiger als Teppich). Die Autoren interpretierten dieses Ergebnis als Evidenz für die Coarse coding Hypothese, da die Metapher mittels einer Überlappung der semantischen Felder von Gans und Verstand aufgelöst werden kann. Jedoch war in diesem Experiment die Plausibilität der nicht-metaphorischen, wörtlichen Sätze viel leichter zu bewerten als die der metaphorischen. Die PFC-Aktivierung könnte allein durch diesen Aufgabenunterschied bedingt sein [vgl. 56].

Kürzlich wurde eine andere Aufgabe verwendet, um diese alternative Erklärung auszuschliessen [57]. Rapp und Kollegen präsentierten nur plausible Sätze, deren affektive Valenz zu bewerten war (positiv oder negativ). Im Vergleich von metaphorischen $\mathrm{zu}$ wörtlichen Interpretationen ergaben sich ausschließlich linksseitige fronto-temporale Aktivierungen, die im Einklang mit einer erhöhten semantischen Verarbeitung stehen [vgl. 58].

Ähnlich inkonsistente Ergebnisse wurden für das Verstehen von moralischen Implikationen berichtet. Die klassische Studie verglich verschiedene Aufgaben zum Verstehen von Aesopschen Fabeln [59]. Für eine Bewertung der „Moral von der Geschicht“ wurde mehr fronto-temporale RH-Aktivierung gefunden, als für eine semantische Aufgabe. Neuere Studien konnten diese Ergebnisse jedoch nicht replizieren, sondern bestätigten eine frontomediane Beteiligung auch am moralischen Urteilen [60].

Der dritte Bereich der pragmatischen Sprachnutzung, den die Bildgebung untersucht hat, ist das Verstehen von verbalem $\mathrm{Hu}-$ mor [61]. Zwei Witzarten wurden von Goel und Dolan präsentiert: Die so genannten phonologischen Witze basierten auf einem Wortspiel (z.B. Why does the golfer wear two pairs of pants? Because he's got a hole in one.), die semantischen auf Weltwissen und sozialen Konventionen (z. B. What do engineers use for birth control? Their personalities.). Die subjektiv empfundene Witzigkeit dieser Frage-Antwort-Paare manifestierte sich in ventro- medianer präfrontaler Aktivierung, ähnlich wie sie auch für emotional gefärbte Problemlöseaufgaben [62] und für emotionale Aspekte der Situationsmodellbildung berichtet wurde [51].

In einer unserer Studien versuchten wir, die sprachlichen Anforderungen von Witzen von der affektiven Komponente zu trennen [43]. In dem Golf-Beispiel etwa ist die Interpretation der Phrase hole in one zweideutig. Sie kann sich entweder auf das Niveau des Golfspiels beziehen - wodurch das Satzpaar inkohärent wird - oder wörtlich auf das Loch in einer der Hosen - was jedoch eine nicht-präferierte Lesart des idiomatischen Ausdrucks erfordert. Das Schmunzeln kommt erst dadurch zustande, dass die dominante Lesart revidiert werden muss. In unserem Experiment konstruierten wir ähnlich zu revidierende Texte, die aber nicht als witzig empfunden wurden. Eine Kontrollbedingung bestand aus Geschichten, die auch ohne Revision kohärent waren. Ein Vergleich der Revisionsgeschichten mit dieser Kontrollbedingung sollte Aufschluss über die neuronale Entsprechung der linguistischen Revision geben, ein Vergleich der Witze mit den Revisionsgeschichten über die zusätzliche affektive Komponente. Im Vergleich aller Textarten mit einer inkohärenten Kontrollbedingung wurde die fronto-mediane Beteiligung an Inferenzprozessen repliziert. Revisionsprozesse führten zu verstärkter Aktivierung im posterioren zingulären Kortex (PCC), einer Region, die mit der Nutzung von Weltwissen und dem Updating von Situationsmodellen in Verbindung gebracht wurde $[38,63]$. Die Witzigkeit der Geschichten stand in Zusammenhang mit einer Aktivierung im ventro-medianen präfrontalen Kortex, eine Replikation der vorher erwähnten Ergebnisse [61].

Zusammenfassend zeigen diese Befunde zu pragmatischen Aspekten ebenfalls Hinweise sowohl auf rechts-hemisphärische als auch auf frontale Beiträge zum Sprachverstehen im Kontext. Die beiden Experimente zu metaphorischer Sprache illustrieren, wie wichtig die Aufgabenstellung ist und legen eine RessourcenInterpretation der RH-Hypothese nahe. Vor allem aber kristallisierte sich einmal mehr die Bedeutung medianer Areale heraus. Der ventrale Anteil des medianen präfrontalen Kortex scheint in Zusammenhang mit den affektiv-pragmatischen, eher unbewussten Sprachprozessen zu stehen, während der dorsale wiederum auftrat, wenn Inferenzen oder Plausibilitätsurteile erforderlich waren, also eine bewusste kontemplative Bewertung vorgenommen wurde.

\section{Fazit}

Auf den ersten Blick scheint diese Zusammenfassung bildgebender Studien möglicherweise mehr ungelöste Fragen offen gelegt, als eindeutige Antworten geliefert zu haben. Eine Beteiligung vieler Hirnregionen ist jedoch nicht überraschend, wenn man die eingangs zusammengefasste Vielfalt der involvierten Prozesse und ihrer Interaktionen mit spezifischen Aufgabenstellungen berücksichtigt. Wesentlicher ist jedoch die Übereinstimmung zwischen den Studien. Vor allem das „erweiterte Sprachnetzwerk“, wie oben beschrieben [33,34,36,37,62], wurde mit einer Reihe verschiedener Methoden, Materialien und Aufgabenstellungen repliziert und kann daher als gesichert gelten [16,32]. Zum Abschluss evaluieren wir die eingangs skizzierten Theorien 
und diskutieren mögliche Funktionen der für die Textverarbeitung einzelner Hirnareale.

\section{Die rechte Hemisphäre}

Eine Beteiligung der rechten Hemisphäre an der Sprachverarbeitung im Kontext steht außer jedem Zweifel, unterstützt sowohl durch Patientenstudien als auch durch Ergebnisse aus der Bildgebung. Jedoch sind die Ergebnisse nicht einheitlich. Zu fast jeder Fragestellung gibt es Studien, die eine Beteiligung rechtsseitiger Hirnareale berichten, und andere, die vor allem linksseitige fronto-temporale und mediane Strukturen aktiviert finden. Gründe dafür könnten methodisch bedingt sein, da die rechte Hemisphäre anatomisch variabler ist als die linke. Positive RHErgebnisse werden häufig mit Region-of-Interest Analysen erzielt, die toleranter gegenüber einer breiteren örtlichen Streuung der individuellen Aktivierungen sind (z. B. [46] vs. [34] zu Inferenzen). Wegweisender für zukünftige Forschung ist jedoch die Vermutung, dass die experimentellen Paradigmen und Materialien unterschiedliche Teilprozesse ansteuern (z. B. [55] vs. [57] zum Metaphern-Verständnis). Künftig wird man die Aufgabenanforderungen und den Anteil affektiver Komponenten sorgfältiger kontrollieren müssen, so dass sich die Hypothesen auf eine der eingangs skizzierten Theorien stützen lassen (z. B. Ressource-Theorie vs. Coarse Coding). Außerdem müssen die postulierten Teilprozesse spezifisch auf Subregionen innerhalb der rechten Hemisphäre abgebildet werden.

\section{Der laterale präfrontale Kortex}

Da Textverstehen und Pragmatik höhere kognitive Leistungen sind, die sowohl syntaktische und semantische Prozesse, als auch exekutive Funktionen beinhalten, ist die Vorhersage massiver Beiträge des präfrontalen Kortex offensichtlich. Auch die Beobachtung nicht-aphasischer Kommunikationsstörungen nach frontalen Läsionen legt einen klaren Zusammenhang nahe. Bei der Evaluation der bildgebenen Studien fällt jedoch auf, dass die frontalen Beteiligungen sehr unterschiedlich ausfallen (z. B. [64] - für nur minimale PFC Aktivierung während des Verstehens von Geschichten). Daher ist bei einer Bewertung immer notwendig, eine genaue Aufgabenanalyse vorzunehmen, und vor allem auch die Kontrollbedingung dabei im Blick zu behalten. Inzwischen ist die funktionelle Neuroanatomie des Frontalhirns so gut erforscht, dass sich frontale Aktivierungen ziemlich genau qualitativ unterschiedlichen Teilprozessen zuordnen lassen. Je nach Lokalisation ist Aktivierung innerhalb des inferioren frontalen Gyrus [s. 30] ein Hinweis auf phonologische und syntaktische Verarbeitung (BA 44), semantische Auswahl (BA 45), oder gedächtnis-basierte Entscheidungen (BA 47/11). Exekutive Fuktionen, wie Inhibition, Sequenzierung oder die Integration widersprüchlicher Information führt zu Beteiligung des posterioren präfrontalen Kortex (BA 9/46; vgl. [56]), und Arbeitsgedächtnisoder Aufmerksamkeitsprozesse benötigen den mittleren frontalen Gyrus [65]. Diese Zuordnungen sind hier natürlich etwas vereinfacht, bieten aber eine gute Basis für das Verständnis frontaler Aktivierungen im Zusammenhang mit Textverständnisaufgaben. Wichtig ist aber, dass Kommunikationsstörungen, die mit Läsionen in diesen Arealen einhergehen, auch Störungen in den jeweiligen basalen Prozessen wahrscheinlich machen.

Zusätzlich zu diesen schon durch die neuropsychologischen Theorien motivierten Bereichen, führten die bildgebenden Studi- en zu einer besonderen Berücksichtigung von zwei weiteren Arealen, deren spezifische Funktion für die Sprachverarbeitung im Kontext aus Läsionsstudien noch weniger bekannt war.

\section{Der anteriore Temporallappen}

Ein unerlässlicher Teil des erweiterten Sprachnetzwerks ist der anteriore Temporallappen. Funktionelle Zuordnungen anterior temporaler Regionen schliessen semantische Prozesse ein, insbesondere die kategorienspezifische Verarbeitung [66]. Wegen der temporo-medial gelegenen Hippokampusformation und der Amygdala spielt der anteriore Temporallappen auch für episodisches und autobiographisches Gedächtnis, sowie emotionale Prozesse eine Rolle [67]. In den Studien zum Textverstehen liegen die Aktivierungen jedoch meist lateral, am vorderen Ende des Sulcus temporalis superior [34,68]. Da eine Beteiligung nicht nur auf Textebene, sondern schon bei Satzverständnisaufgaben beobachtet wird [36,37,62], und da sie sprachspezifisch scheint [69], weisen wir dem anterioren Temporallappen vorläufig eine Rolle für die Propositionalisierung zu [8]. Dieses Übertragen der wörtlichen Formulierung in Inhaltseinheiten erfordert ein $\mathrm{Zu}$ sammenspiel syntaktischer, semantischer und episodischer Prozesse. Auch hier ist natürlich weitere Forschung nötig, um zu klären, unter welchen Bedingungen auch satzübergreifende Verarbeitung diese Region erfordert. Ausserdem ist nicht bekannt, wie die meist gemeinsam aktivierten links- und rechtshirnigen anterioren temporalen Regionen zusammenspielen.

\section{Der fronto-mediane Kortex}

Der wohl interessanteste Befund ist die konsistente Beteiligung des fronto-medianen Kortex an der Sprachverarbeitung im Kontext. Im Gegensatz zu Aktivierung des ventralen fronto-medianen Kortex (BA 11), die als Hinweis auf affektive Aspekte interpretiert wurde $[51,61,70]$, steht die Aktivierung des dorsalen fronto-medianen Kortex in Zusammenhang mit Inferenzprozessen. Diese Region umfasst Brodmann-Areale 8, 9 und 10 und wird vom darunter liegenden anterioren zingulären Kortex (ACC, BA 24) unterschieden. Die Vorschläge für die Funktionalität dieses Areals reichen von emotionaler Verarbeitung, über selbst-relevante Prozesse bis zu so genannten Theory-of-Mind (ToM) Prozessen [33]. Obwohl jede Kommunikation relevant für das Selbst ist, und eine Interaktion mit dem Gesprächspartner immer ToM einschließt [71], postulieren wir einen domänen-übergreifenden Prozess, der sowohl Inferenz-, Evaluierungs-, Entscheidungs-, und ToM-Prozesse beinhaltet [33]. Charakteristisch für diesen Prozess ist, dass er nicht-automatisch, wissensbasiert und durch einen individuellen Lösungsweg gekennzeichnet ist. Die Wissenskomponente dieses Prozesses spiegelt sich häufig in einer gleichzeitigen Aktivierung des parieto-medianen Kortex wider (Präcuneus, posteriorer zingulärer Kortex, BA 31), einer Region, deren Funktion spezifisch mit der Integration neuer Information ins Situationsmodell beschrieben wurde [35,38].

\section{Klinische Implikationen}

Durch die Ergebnisse dieser Studien wird bestätigt, dass Sprachverstehen im Kontext weit mehr erfordert als die Beteiligung des perisylvischen Sprachkortex. Die fronto-temporalen Regionen des erweiterten Sprachnetzwerks können je nach Aufgabenanforderungen durch den gesamten kognitiven Apparat unterstützt werden. Läsionen in diesem Netzwerk führen dazu, dass Patienten ohne basale Sprachdefizite dennoch Auffälligkeiten in der 
Kommunikation zeigen. Umgekehrt erklären die ausgedehnten Beiträge nicht-primär sprachrelevanter kortikaler Areale, dass aphasische Patienten in der Kommunikation oft erfolgreicher sind, als dies der Schweregrad ihrer Defizite vorhersagen ließe.

Die Bildgebung hat hierbei entscheidend zu unserem Verständnis beigetragen. Sowohl das RH-Syndrom [15], das ja eine kommunikative Problematik einschließt, als auch eine kommunikative Einschränkung als Folge einer exekutiven Funktionsstörung werden durch die Bildgebung gestützt. Zusätzlich zu dieser teilweisen Bestätigung der aus der klinischen Neuropsychologie abgeleiteten Theorien wurden Hirnareale identifiziert, die bisher nicht spezifisch in Läsionsstudien zum Sprachverstehen im Kontext thematisiert wurden: der anteriore Temporallappen beidseits und der dorsale fronto-mediane Kortex. Neben diffuser axonaler Schädigung erzeugen Kontusionsverletzungen bei SchädelHirn-Trauma (SHT) besonders häufig Läsionen auch dieser Hirnregionen [72]. Häufig beobachtete nicht-aphasische Kommunikationsdefizite nach SHT können somit durch Ergebnisse aus der Bildgebung mit begründet werden.

Zusammengefasst bestätigen die hier besprochenen Studien einmal mehr, dass für alle Patienten mit Schädel-Hirn-Trauma und Patienten mit Frontalhirn- und rechts-hemisphärischen Läsionen anderer Ätiologien eine differenzierte Diagnostik der Kommunikationsfähigkeiten angezeigt ist. Eine Bewertung der alltagsrelevanten Kommunikationsfähigkeiten erfordert dabei immer sowohl die Berücksichtigung aphasischer Symptome als auch eine Diagnostik von Textverständnis, Diskursproduktion und affektiv-pragmatischen Aspekten der Sprachnutzung im Kontext.

\section{Literatur}

${ }^{1}$ Brooks N, McKinlay W, Symington C et al. Return to work within the first seven years of severe head injury. Brain Inj 1987; 1: 5-19

${ }^{2}$ Kintsch W. Comprehension: A paradigm for cognition. Cambridge: Cambridge University Press, 1998

${ }^{3}$ Glindemann R, von Cramon DY. Kommunikationsstörungen bei Patienten mit Frontalhirnläsionen. Sprache, Stimme, Gehör 1995; 19: $1-7$

4 Prigatano GP, Roueche JR, Fordyce DJ. Nonaphasic language disturbances after brain injury. In: Prigatano GP, Fordyce DJ, Zeiner HK, Roueche JR, Pepping M, Wood BC (Hrsg). Neuropsychological rehabilitation after brain injury Baltimore, MD: John Hopkins University Press, 1986: $18-28$

${ }^{5}$ American Speech-Language-Hearing Association. The role of the speech-language pathologists in the identification, diagnosis, and treatment of individuals with cognitive-communicative impairments. ASHA, 1988: 30-79

${ }^{6}$ Graesser AC, Singer M, Trabasso T. Constructing inferences during narrative text comprehension. Psychol Rev 1994; 101: 371 - 395

${ }^{7}$ Singer M. Discourse inference processes. In: Gernsbacher MA (Hrsg): Handbook of psycholinguistics. San Diego, CA: Academic Press, 1994: 479-515

${ }^{8}$ Kintsch W, van Dijk TA. Toward a model of text comprehension and production. Psychol Rev 1978; 85: 363-394

9 van Dijk TA, Kintsch W. Strategies of discourse comprehension. New York: Academic Press, 1983

${ }^{10}$ Ferstl EC, Kintsch W. Learning from text: Structural knowledge assessment in the study of discourse comprehension. In: Oostendorp $\mathrm{H}$, Goldman S (Hrsg). The construction of mental models during reading Mahwah, NJ: Lawrence Erlbaum, 1999: 247-277

${ }^{11}$ Rinck M. Situationsmodelle und das Verstehen von Erzähltexten: Befunde und Probleme. Psychologische Rundschau 2000; 51: 115-122
12 Zwaan RA, Magliano JP, Graesser AC. Dimensions of situation model construction in narrative comprehension. J Exp Psychol Learn Mem Cog 1995; 21: $386-397$

${ }^{13}$ Ferstl EC. Learning from Text. In: Smelser NJ, Baltes PB (Hrsg). International Encyclopedia of the Social \& Behavioral Sciences, Volume 3.13: Cognitive Psychology and Cognitive Science (Ed. Kintsch W) Amsterdam: Elsevier, 2001

${ }^{14}$ Mey JL. Pragmatics: An introduction. Oxford, GB: Blackwell, 2001

15 Brownell HH, Martino G. Deficits in inference and social cognition: The effects of right hemisphere brain damage on discourse. In: Beeman M, Chiarello C (Hrsg). Right hemisphere language comprehension: Perspectives from cognitive neuroscience Mahwah, NJ: Lawrence Erlbaum, 1998: 309-328

${ }^{16}$ Ferstl EC (im Druck). The functional neuroanatomy of text comprehension: What's the story so far? In: Schmalhofer F, Perfetti CA (Hrsg). Higher Level Language Processes in the Brain: Inference and Comprehension Processes Lawrence Erlbaum,

${ }^{17}$ Springer SP, Deutsch G. Left brain, right brain. New York: Freeman, 1997

${ }^{18}$ Navon D. Resources: A theoretical soup stone? Psychol Rev 1984; 91 : $206-234$

19 Beeman M. Coarse semantic coding and discourse comprehension. In: Beeman M, Chiarello C (Hrsg). Right hemisphere language comprehension: Perspectives from cognitive neuroscience Mahwah, NJ: Lawrence Erlbaum, 1998: 255-284

${ }^{20}$ Kintsch W. The use of knowledge in discourse processing: A construction-integration model. Psychol Rev 1988; 95: 163-182

${ }^{21}$ Lehmann MT, Tompkins CA. Inferencing in adults with right hemisphere brain damage: an analysis of conflicting results. Aphasiology 2000; 14 (5/6): $485-499$

${ }^{22}$ Ferstl EC, Guthke T, von Cramon DY. Change of perspective in discourse comprehension: Encoding and retrieval processes after brain injury. Brain Lang 1999; 70: 385-420

${ }^{23}$ Novoa OP, Ardila A. Linguistic abilities in patients with pre-frontal damage. Brain Lang 1987; 30: 206-225

${ }^{24}$ Ettlin TM, Beckson M, Gaggiotti M et al. The frontal lobe score: part I: construction of a mental status of frontal systems. Clin Rehabil 2000; 14: $260-271$

25 Damasio A. Descartes' Error. London: Picador, 1995

${ }^{26}$ Luan Phan K, Wager T, Taylor SF et al. Functional neuroanatomy of emotion: A meta-analysis of emotion activation studies in PET and fMRI. NeuroImage 2002; 16: $331-348$

${ }^{27}$ Ferstl EC, Guthke T, von Cramon DY. Text comprehension after brain injury: Left prefrontal lesions affect inference processes. Neuropsychology 2002; 16: $292-308$

${ }^{28}$ McDonald S. Viewing the brain sideways? Frontal versus right hemisphere explanation of non-aphasic language disorders. Aphasiology 1993; 7: 535-549

${ }^{29}$ Nicholas LE, Brookshire RH. Comprehension of spoken narrative discourse by adults with aphasia, right-hemisphere brain damage, or traumatic brain injury. Am J Speech Lang Pathol 1995; 4: 69-81

${ }^{30}$ Bookheimer S. Functional MRI of language: new approaches to understanding the cortical organization of semantic processing. Annu Rev Neurosci 2002; 25: $151-188$

${ }^{31}$ Gernsbacher MA, Kaschak MP. Neuroimaging studies of language production and comprehension. Annu Rev Psychol 2003; 54: 91 - 114

32 Mar RA. The neuropsychology of narrative: story comprehension, story production and their interrelation. Neuropsychologia 2004; 42: $1414-1434$

${ }^{33}$ Ferstl EC, von Cramon DY. What does the fronto-medial cortex contribute to language processing: Coherence or Theory of Mind? NeuroImage 2002; 17: 1599-1612

${ }^{34}$ Ferstl EC, von Cramon DY. The role of coherence and cohesion in text comprehension: an event-related fMRI study. Brain Res Cogn Brain Res 2001; 11: $325-340$

${ }^{35}$ Fletcher PC, Happe F, Frith U et al. Other minds in the brain: A functional imaging study of "theory of mind“ in story comprehension. Cognition 1995; 57: 109-128

${ }^{36}$ Mazoyer BM, Tzourio N, Frak V et al. The cortical representation of speech. J Cogn Neurosci 1993; 5: 467-479

${ }^{37} \mathrm{Xu}$ J, Kemeny S, Park G et al. Language in context: emergent features of word, sentence, and narrative comprehension. Neurolmage 2005; 25 : $1002-1015$ 
${ }^{38}$ Maguire EA, Frith CD, Morris RGM. The functional neuroanatomy of comprehension and memory: The importance of prior knowledge. Brain 1999; 122: 1839-1850

${ }^{39}$ Binder JR, Frost JA, Hammeke TA et al. Human brain language areas identified by functional magnetic resonance imaging. J Neurosci1997; 171: $353-362$

${ }^{40}$ Scott SK, Leff AP, Wise RJS. Going beyond the information given: a neural system supporting semantic interpretation. NeuroImage 2003; 19: $870-876$

${ }^{41}$ Binder JR, Frost JA, Hammeke TA et al. Conceptual processing during the conscious resting state: a functional MRI study. J Cogn Neurosci 1999; 11: 80-93

${ }^{42}$ Siebörger FT, Ferstl EC, von Cramon DY. Making sense of nonsense: An fMRI study of task induced coherence processes. J Int Neuropsychol Soc 2003; 9: 506

${ }^{43}$ Siebörger FT, Ferstl EC, von Cramon DY. Is linguistic revision necessary for the appreciation of verbal humor? An fMRI study on the comprehension of jokes. Manuscript in preparation, 2004

${ }^{44}$ Schmalhofer F, Raabe M, Friese U et al. Evidence from an fMRI experiment for the minimal encoding and subsequent substantiation of predictive inferences. Proceedings of the 26th Meeting of the Cognitive Science Society, 2004

${ }^{45}$ Robertson DA, Gernsbacher MA, Guidotti SJ et al. Functional neuroanatomy of the cognitive process of mapping during discourse comprehension. Psychol Sci 2000; 11: 255-260

${ }^{46}$ Mason RA, Just MA. How the brain processes causal inferences in text: A theoretical account of generation and integration component processes utilizing both cerebral hemispheres. Psychol Sci 2004; 15: 1 - 7

${ }^{47}$ Caplan R, Dapretto M. Making sense during conversation: an fMRI study. NeuroReport 2002; 12: 3625 - 3632

48 Bransford JD, Johnson MK. Contextual prerequisites for understanding: Some investigations of comprehension and recall. Journal of Verbal Learning and Verbal Behavior 1972; 11: 717-726

${ }^{49}$ St. George M, Kutas M, Martinez A et al. Semantic integration in reading: Engagement of the right hemisphere during discourse processing. Brain 1999; 122: $1317-1325$

50 Dooling DJ, Lachman R. Effects of comprehension on retention of prose. J Exp Psychol 1971; 88: 216 - 222

${ }^{51}$ Ferstl EC, Rinck M, von Cramon DY. Emotional and temporal aspects of situation model processing during text comprehension: An event-related fMRI study. J Cogn Neurosci 2005; 17: 724-739

52 Rinck M, Hähnel A, Becker G. Using temporal information to construct, update, and retrieve situation models of narratives. J Exp Psychol Learn Mem Cog 2001; 27: 67-80

${ }^{53}$ Walsh V. A theory of magnitude: common cortical metrics of time, space and quantity. Trends Cogn Sci 2003; 7: 483-488
${ }^{54}$ Zwaan RA. The immersed experiencer: Toward an embodied theory of language comprehension. In: Ross BH (Hrsg). The Psychology of Learning and Motivation; Vol. 44 Amsterdam: Elsevier, 2004: 35-62

${ }^{55}$ Bottini G, Corcoran R, Sterzi R et al. The role of the right hemisphere in the interpretation of figurative aspects of language: a positron emission tomography activation study. Brain 1994; 117: 1241 - 1253

${ }^{56}$ Derrfuss J, Brass M, von Cramon DY. Cognitive control in the posterior frontolateral cortex: Evidence from common activations in task coordination, interference control, and working memory. Neurolmage 2004; 23: 604-612

${ }^{57}$ Rapp AM, Leube DT, Erb M et al. Neural correlates of metaphor processing. Brain Res Cogn Brain Res 2004; 20: 395-402

${ }^{58}$ Glucksberg S. The psycholingustics of metaphor. Trends Cogn Sci 2003; 7: $92-96$

${ }^{59}$ Nichelli P, Grafman J, Pietrini P et al. Where the brain appreciates the moral of a story. NeuroReport 1995; 6: 2309-2313

${ }^{60}$ Heekeren HR, Wartenburger I, Schmidt $\mathrm{H}$ et al. An fMRI study of simple ethical decision-making. NeuroReport 2003; 14: 1215-1219

${ }^{61}$ Goel V, Dolan RJ. The functional anatomy of humor: segregating cognitive and affective components. Nature Neuroscience 2001; 4 (3): 237-261

62 Bavelier D, Corina D, Jezzard P et al. Sentence reading - a functional MRI study at 4 Tesla. J Cogn Neurosci 1997; 9: 664-686

${ }^{63}$ Zysset S, Huber O, Ferstl EC et al. The anterior dmPFC and evaluative judgment: An fMRI study. NeuroImage 2002; 15: 983 -991

${ }^{64}$ Crinion JT, Lambon-Ralph A, Warburton EA et al. Temporal lobe regions engaged during normal speech comprehension. Brain 2003; 126: $1193-1201$

${ }^{65}$ Gruber O. Effects of domain-specific interference on brain activation associated with verbal working memory task performance. Cereb Cortex 2001; 11: $1047-1055$

${ }^{66}$ Martin A, Chao LL. Semantic memory and the brain: structure and processes. Curr Opin Neurobiol 2001; 11: 194-201

${ }^{67}$ Maratos EJ, Dolan RJ, Morris JS et al. Neural activity associated with episodic memory for emotional context. Neuropsychologia 2001; 29: $910-920$

68 Perani D, Dehaene S, Grassi F et al. Brain processing of native and foreign languages. Neuroreport 1996; 7: 2439-2444

${ }^{69}$ Humphries C, Willard K, Buchsbaum B et al. Role of anterior temporal cortex in auditory sentence comprehension: an fMRI study. Neuroreport 2001; 12: 1749-1752

70 Goel V, Dolan RJ. Reciprocal neural response wihtin lateral and ventral medial prefrontal cortex during hot and cold reasoning. Neurolmage 2004; 20: 2314-2321

${ }^{71}$ Frith U, Frith CD. Development and neurophysiology of mentalizing. Philos Trans R Soc Lond B Biol Sci 2003; 358: 459-473

72 Adams RD, Victor M. Principles of Neurology. New York, NY: McGrawHill, 1993 